\title{
Review and Application of Model and Spectral Analysis Based Fault Detection and Isolation Scheme in Actuators and Sensors
}

\author{
H. Bal ${ }^{2}$, S. K. Mohanty ${ }^{1}$, N. P. Mahalik ${ }^{2, *}$, B. B. Biswal ${ }^{3}$ \\ ${ }^{1}$ Colllege of Engineering, Biju Patnaik University of Technology, Bhubaneswar, Odisha, India \\ ${ }^{2}$ Department of Industrial Technology, Jordan College of Agricultural Sciences and Technology, California State University, Fresno, \\ California, USA \\ ${ }^{3}$ National Institute of Technology, Rourkela, India
}

Email address:

skmohanty03@yahoo.com (S. K. Mohanty),nmahalik@gmail.com (N. P. Mahalik), bibhuti.biswal@gmail.com (B. B. Biswal)

\section{To cite this article:}

H. Bal, S. K. Mohanty, N. P. Mahalik, B. B. Biswal. Review and Application of Model and Spectral Analysis Based Fault Detection and Isolation Scheme in Actuators and Sensors. Automation, Control and Intelligent Systems. Vol. 3, No. 4, 2015, pp. 49-55.

doi: 10.11648/j.acis.20150304.11

\begin{abstract}
For condition monitoring of machineries and systems conventional method such as hardware or sensor based error checking scheme were in use. As the automated systems are becoming complex, recently most of the condition-monitoring schemes have been applying sophisticated analytical tools and methods to achieve improved performance. The objective of this paper is to demonstrate model based Fault Detection and Isolation (FDI) schemes for mechatronic systems and devices. First we have reviewed FDI approaches and implementation schemes. Then, we have developed two frameworks: model and spectral signature based for the implementation of FDI schemes. The model based feature estimation and spectral analysis based multiresolution methods are implemented in exemplar devices such as actuators and sensors used in mechatronic systems. Based on the frameworks, the diagnostics and isolation algorithms were developed using MATLAB code. The algorithms are capable of detecting and isolating faults within the systems. The study is comprehensive and the implementation scenarios can be extendible to many types of systems and devices used in the mechatronic domain.
\end{abstract}

Keywords: FDI, Model-Based, Spectral Analysis, Multiresolution, ANN, FL

\section{Introduction}

For condition monitoring of machineries and systems, conventional method such as hardware based error checking $[1,15]$ has been very popular since long. As the automated systems are becoming complex, recently most of the condition-monitoring schemes have been applying sophisticated analytical tools and methods to achieve improved performance [2]. Review on various condition monitoring and FDI approaches can be found from [3]. In this paper we have presented sophisticated analytical methods and approaches in order to develop FDI algorithms which can be applied to a range of systems and devices. A brief review on model-based FDI technique for actuating systems is presented below. Primarily, the model-based approach prepares a model that facilitates generating residuals which are measured and tested statistically in order to compare with the logical normal patterns for decision making of fault symptoms as illustrated in the Fig. 1 [16-17].

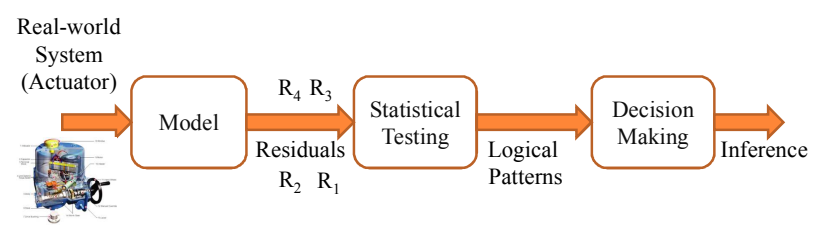

Figure 1. Stages involved in model based FDI approach.

Mechatronic systems are composed of actuators and sensors. We have applied model-based technique to actuators and multi-resolution spectral analysis methods to sensors. The objective is to detect faults in the motor during its operation. First, we reviewed a framework to define appropriate model equations in order to accommodate features capable of finding faults. Fig. 2 shows the schematic of model based FDI scenario. For detail derivation, refer [2-5]. Here, $\mathrm{A}(\mathrm{s})$ and $\mathrm{B}(\mathrm{s})$ are system matrix, $\mathrm{u}$ and $\mathrm{y}$ are 
inputs and outputs respectively. The feature estimation process is based on a task that determines the dependencies between different measurable signals - expressed by mathematical models. Based on measured input/output signals the detection methods generate residuals and then estimate the feature for comparison with the normal features. The changes of features are detected and faults are isolated.

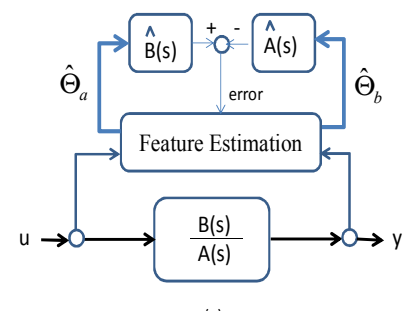

(a)

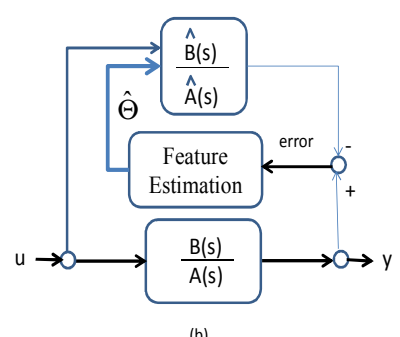

(b)
Figure 2. Feature estimation method with minimum errors (a) Equation error, (b) Output error $[3,15,17]$.

\section{Feature Estimation Based FDI for Actuation Valve Driven by Motor}

In this experimental study, we developed a prototype to replicate the motor-actuation based valve control system as shown in Fig. 3. The main valve has two openings: left side opening and right side opening. There are two motors. Each motor drives its corresponding auxiliary valve which is interfaced with the main valve to either open the left side or right side based on the control need. This each motor drives the outflow valve either in positive or negative direction depending upon the amount of opening and closing need. The entire control strategy was implemented based on Distributed Control Scheme (DCS). Under this scenario

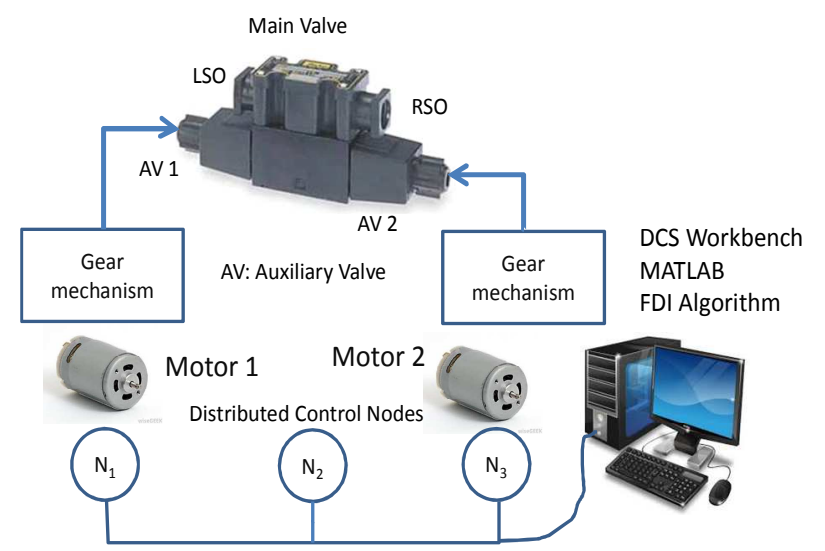

Figure 3. The prototype of motor-actuation based valve control system.

In order to detect the fault it was required to identify the feasible measurands. For this system, the considered measurands are $V(t), I(t), \omega_{r}(t)$, and $\theta_{g}(t)$. These measurands can be segmented to auxiliary measurands. For example, considering time variant current, the voltage equation of the electrical subsystem can be written $\mathrm{V}(\mathrm{t})-\psi_{E}$
$\omega_{r}(t)=\mathrm{RI}(t) ; \mathrm{R}$ is the resistance of the coil and $\psi_{E}$ flux linkage. Thus, flux is derived to be one of the auxiliary measurands featuring faults because the generated rotor torque is proportional to the effective magnetic flux linkage. Note that $\psi_{T}<\psi_{E}$ and is given by $\mathfrak{I}_{r}(t)=\psi_{T} \mathrm{I}(\mathrm{t})$. Similarly, the dynamic equation of the motor can be written as $J_{r} \omega_{r}(t)=\psi_{T} I(t)-\mathfrak{I}_{f}(t)-\mathfrak{I}_{L}(\mathrm{t})$, where $\mathrm{J}$ is called the moment of inertia. The friction torque becomes $\mathfrak{I}_{f}(t)=c_{f} \operatorname{sign} \omega_{r}(t)$ and load torque is $\mathfrak{I}_{L}(t)$. The expression $\theta_{g}=\theta_{r} / v$ is the relationship between the gear ratio $v$ and shaft position $\left(\theta_{r}\right)$ and flap position $\left(\theta_{g}\right)$. The value of $v$ from the data sheet is 1294 . Now the torque of the flap becomes $\mathfrak{I}_{L}=c_{s} f\left(\theta_{g}\right)$. Let us formulate a model equation that can reflect the feature estimation scheme. A suggested formulation can be $y(t)=\Delta^{T}(t) \theta$. The following expressions are written to describe the residuals; each is coupled from at least one measurands [3]. Respective residuals are considered as the image of the respective faults [5]. Several residuals can be combined to represent some form of composite faults. The important variables that are considered for measurement are voltage V; current I, friction $c_{f}$, resistance $\mathrm{R}$, and position $=\phi[2-5]$.

$$
\begin{aligned}
F_{R 1}(t) & =V(t)-R I(t)-\psi_{E} \omega_{r}(t) \\
F_{R 2}(t) & =\psi_{T} I(t)-J_{r} \omega_{r}(t)-c_{f} \operatorname{sign} \omega_{r}(t)-c_{s} f\left(\theta_{g}\right) \\
F_{R 3}(t) & =V(t)-R / \psi_{T}\left(J_{r} \omega_{r}(t)+c_{s} f\left(\theta_{g}\right)\right. \\
& \left.+c_{f} \operatorname{sign} \omega_{r}(t)+\psi_{E} \omega_{r}(t)\right) \\
F_{R 4}(t) & =\theta_{g}(t)-\theta_{r}(t) / v
\end{aligned}
$$

\subsection{Results}

Previously we had implemented the FDI algorithm in DSP (Digital Signal Processor) platform, a centralized system, and presented the results in [4]. This work is a step forward to earlier work in that the control architecture and strategy have been implemented based on DCS (Distributed Control Systems) scheme rather than centralized. That is the FDI algorithm was embedded within the DCS architecture using fieldbus type technology. Once the model is developed, the feature estimation algorithm is written to identify abnormality in the features or parameters. In order to achieve this, two sub-processes are needed: classification and decision. ANN (Artificial Neural Network) based classification is very popular for nonlinear systems. The decision algorithm was implemented using FL (Fuzzy Logic) methods. Fig. 4 illustrates the FDI processes that include both learning and run-time phase.

Fig.5. shows the signals measured at the outputs using sensors. It also shows the feature estimations and the derived residuals. The residuals are estimated based on the equations presented above. Five different faults corresponding to resistance, friction, voltage, position, and current were deliberately incorporated into the system and subsequently 
some of the features were observed via estimation using the model equations. Finally, the fault signatures such as residuals were generated to isolate and localize the exact fault. Some of the experimental results are shown in Table 1. We also conducted the success rate of identifying the faults. The success rate in this laboratory based demonstration system was $100 \%$.
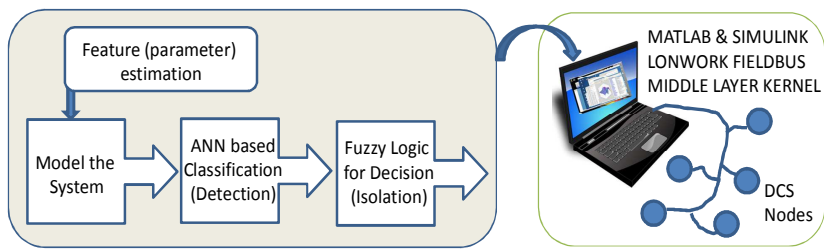

Figure 4. Experimental set up and algorithm development phases.

Table 1. Feature deviation due to faults.

\begin{tabular}{llllllllllll}
\hline \multirow{2}{*}{$\begin{array}{l}\text { Faults } \\
\text { introduced }\end{array}$} & \multicolumn{4}{c}{ Estimation of some features } & \multicolumn{5}{c}{ Residuals generated in } \\
percentage (\%)
\end{tabular}

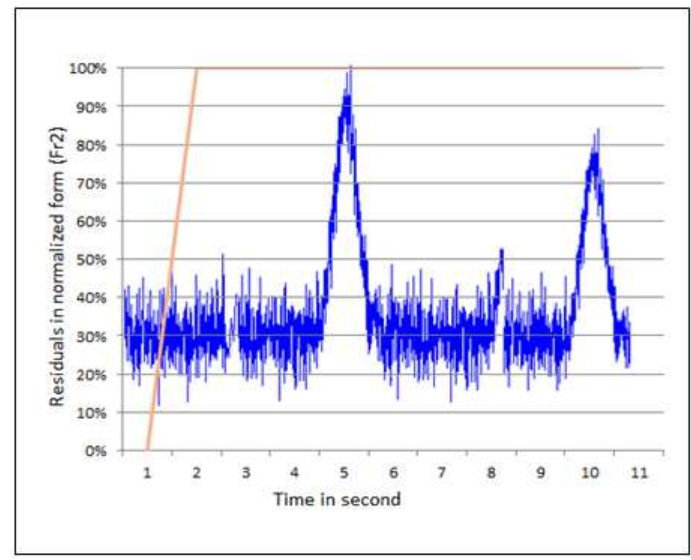

(a)

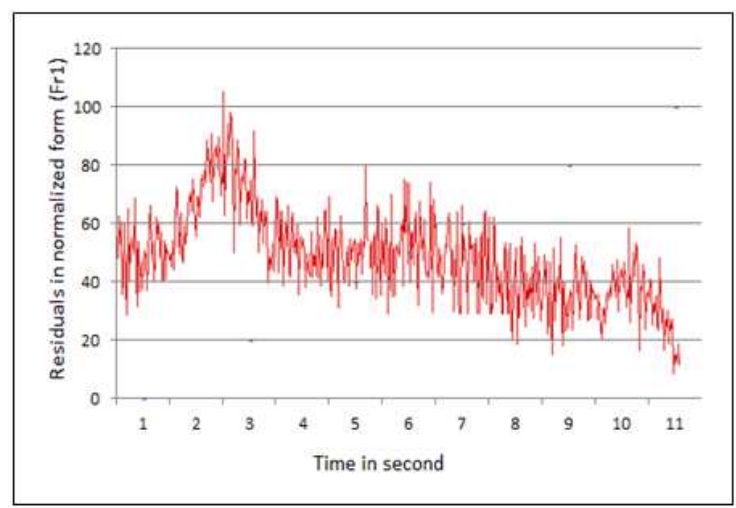

(b)

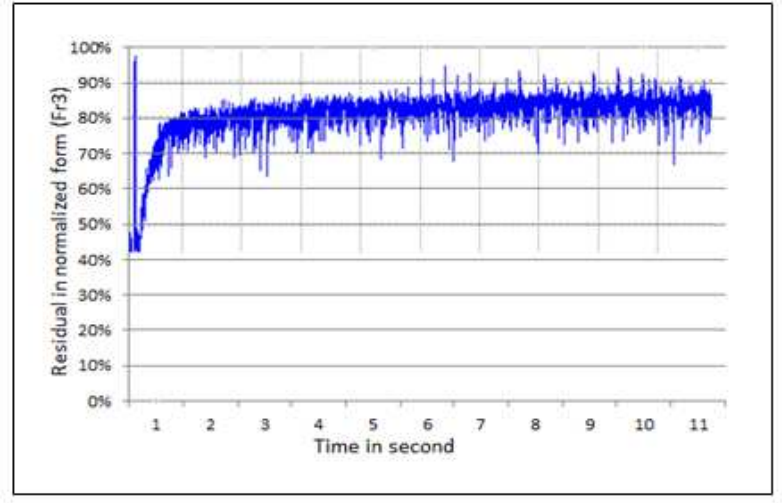

(c)

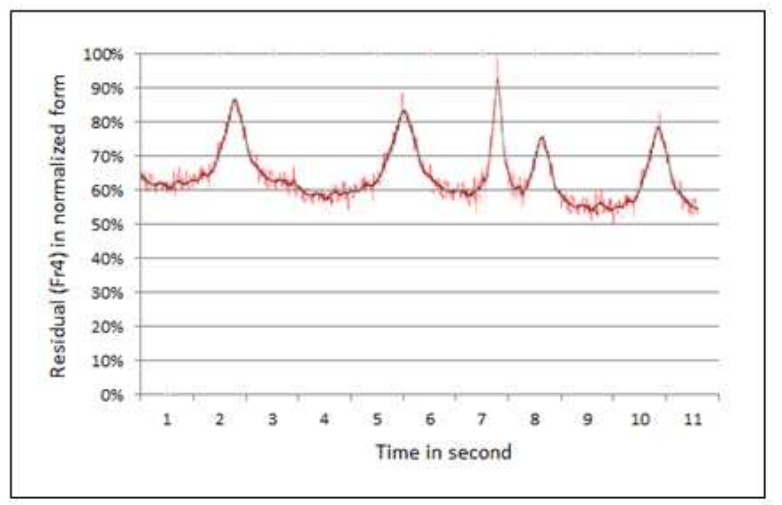

(d)

Figure 5. Feature estimation based FDI results (Residuals when the valve leaks): Residuals at 15-50\% during normal operation. (a) leakage fault, (d) sensor offset positive, (e) sensor offset negative; (B) Residuals for positive offset on supply air.

The estimation in all experiment showed positive $(+)$. Using the normalization method, we have set the threshold value (minimum) of the residual as $75 \%$. The faults were introduced manually. The employed control scheme is closed loop based. Both ANN and FL algorithms) were used to classify [6] and isolate [5] the faults, respectively.

\subsection{Classification and Decision Using $A N N$ and FL}

The FDI phases are described in the Fig. 3. It can be stated that ANN forms the basis of classification, [4] while FL serves as the decision tool. [2]. Both classification and decision together are treated as residual evaluation phases of the FDI [6-7]. Residual evaluation is thus a part of fault isolation [8]. The goal of residual evaluation for FDI is to device whether a fault in a system under consideration has occurred avoiding wrong decision that cause false alarm. In order to reduce the false alarm rate, classification and decision by combination of ANN and FL, respectively are suggested. As a validation of the residual evaluation we conducted several experiments with the above actuator based valve control system. As another example, the Fig. 5 shows some other faults corresponding to appropriate residuals. In particular, it is necessary to understand the leakage fault of 
the valve when it is completely closed. The figure is self-explanatory and the results presented in Fig.4 and Fig.5 are based on the FDI algorithm represented in Fig.3. The classification and decision aspect of the algorithm is realized using ANN and FL toolset of the MATLAB software [9]. The mapping for a given input to an output using FL can be formulated by using a process called fuzzy inference. Fuzzy inference system is having wide range of applications in the fields such as automatic control, data classification, decision analysis, expert system and computer vision [10]. MATLAB has been designed with three functions namely plotfis, plotmf and gensurf which provides a high level view of the fuzzy inference system. All the fuzzy inference system information is contained in the MATLAB object known as FIS (Fuzzy inference system) structure. This structure is stored inside each GUI tool. This structure is examined by access functions such as getfis and setfis.

\section{Second Experiment with Wheelchair}

In considering advancements in computing platform and digital technology, this work studies FDI in actuating systems [11]. Actuators are a class of technological systems that always entail greater reliability. This work presents a study on two types of FDI approaches. They are model-based and spectral analysis.

\subsection{Model-Based FDI}

In the second experiment for the evaluation of the feature estimation based fault detection is a wheel chair shown in the Fig.6. Fig.7. shows schematic of the steering system.

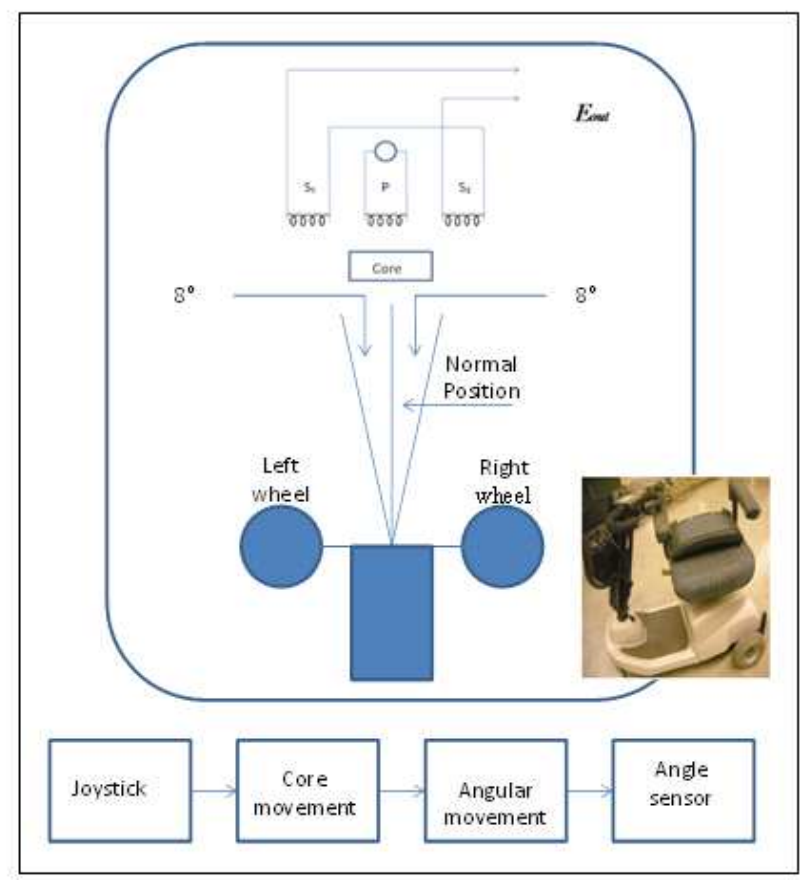

Figure 6. A 3-wheel based rehabilitation chair [18].

The objective is to observe the driving activities while the wheel chair changes the lane. This behavior of the lane change is extremely important to a handicapped person sitting the chair. The faults in the steering actuator must be detected and isolated. If the behavior of the lane change is abnormal then the steering operation must be switched to the standby actuating system.

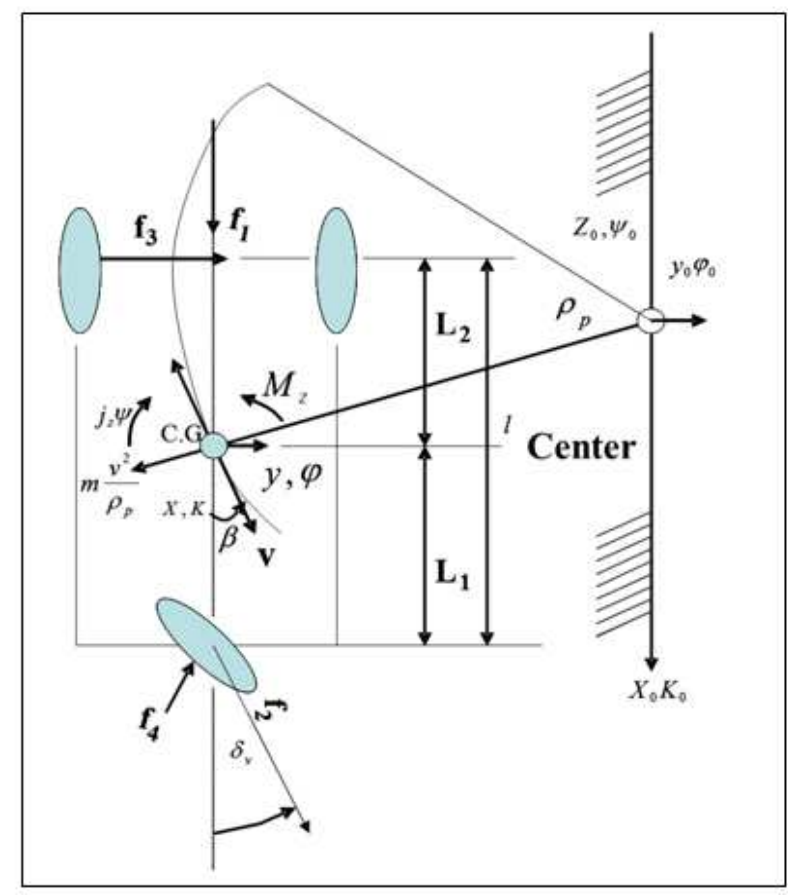

Figure 7. Geometrical drawings of the steering behavior [3].

Based on theoretical modeling it is perceived that the characteristic velocity will be the preferred choice as regards to a feature of this system [3-5]. Here the lane-change behavior is reflected through three behaviors such as normal-steering, under-steering and over-steering. The wheel chair has 3 wheels as shown in the Fig.7. By referring [3], the dynamic model equations of this wheel chair were presented in detail in [5] and [4]. The important model equations used in this paper can be written from [3].

Since this is a lane change scenario, the dynamics can be modeled considering lateral movement. The following terms are defined.

a: position errorb: lateral position error

c: vertical position error $\psi$ : yaw angle

$\Delta:$ steering angle $\Delta a:$ wheel angle

F: slip anglem: mass

$I_{a}$ : moment of inertiaV: driving velocity

$\mathrm{S}_{1}$ : front wheel stiffens $\mathrm{S}_{2}$ : rear wheel stiffness

$\mathrm{L}_{1}$ : front axle length to C.G $\mathrm{L}_{2}$ : rear axle length to C.G

$\mathrm{L}$ : front and rear axle length $\mathrm{i}_{\mathrm{st}}$ : gear ratio

$\rho$ : radius $\mathrm{F}_{1}$ : force acting on rear tire

First, consider the characteristic equation as of a vehicle as follows.

$$
S^{2}+C_{1} S+C_{0}=0
$$

Where, 


$$
\begin{aligned}
& C_{1}=\frac{1}{I_{a} m v}\left[\left(I_{a}+m l^{2}{ }_{1}\right) S_{1}+\left(I_{a}+m l^{2}{ }_{2}\right) S_{2}\right] \\
& C_{0}=\frac{1}{I_{a} m v}\left[S_{1} S_{2}\left(L_{1}+L_{2}\right)^{2}+m v^{2}\left(S_{2} L_{2}-S_{1} L_{1}\right)\right]
\end{aligned}
$$

The characteristic velocity [3] can be written as follows.

$$
V_{c h}^{2}(t)=\frac{S_{1}(t) S_{2}(t)^{2}}{m\left(S_{2}(t) L_{2}-S_{1}(t) L_{1}\right)}
$$

The above characteristic expression that is used for stability is as follows.

$$
\frac{V^{2}}{V_{c h}^{2}}<1
$$

The input and output model equations [3] are as follows.

$$
\begin{aligned}
& {\left[\begin{array}{l}
\ddot{b} \\
\ddot{\psi}
\end{array}\right]=\left[\begin{array}{l}
\frac{s_{1}^{\prime}}{m i_{s t}} \\
\frac{s_{1}^{\prime} L_{1}}{I_{a} i_{s t}}
\end{array}\right] \Delta_{a}=\left[\begin{array}{l}
\frac{m v+s_{2}+s_{1}^{\prime}}{m v} \frac{s_{1} L_{1} s_{1} L_{1}}{m v} \\
\frac{s_{2} L_{2} s_{1}^{\prime} L_{1}}{I_{a} v} \frac{s_{1}^{\prime} L_{1}^{2} s_{2} L_{2}^{2}}{I_{a} v}
\end{array}\right]\left[\begin{array}{l}
\dot{b} \\
\dot{\psi}
\end{array}\right]} \\
& {\left[\begin{array}{l}
\dot{b}(t) \\
\dot{\psi}(t)
\end{array}\right]=\left[\begin{array}{ll}
1 & 0 \\
0 & 1
\end{array}\right]\left[\begin{array}{l}
\dot{b} \\
\dot{\psi}
\end{array}\right]}
\end{aligned}
$$

The steering wheel angle during natural steering will be

$$
\frac{\dot{\psi}(t)}{\Delta a(t)}=\frac{1}{i_{s t} L} \frac{v(t)}{1+\left(\frac{v(t)}{v_{c h}(t)}\right)^{2}}
$$

With the measured steering wheel angle at the input, the velocity and the yaw rate at the output the characteristics velocity can be written as follows.

$$
\left[V_{c h}{ }^{2}(t)\right]\left[1-\frac{\Delta a(t) V(t)}{\dot{\psi}(t) i_{s t} L}\right]=-V^{2}(t)
$$

\subsection{Results}

The overall objective of this research was to develop advanced diagnostic and prognostic method by making the systems more predictable and available through developing model-based dynamic health management algorithms. The developed algorithm utilizing model equations to estimate the features [12] (both variables and parameters) by using sensor signals and refining it to a data infrastructure for FDI usage based in DCS implementation strategy. Thus the research not only demonstrates FDI scheme, but its implementation methods using advanced fieldbus type technology systems and architecture (e.g., DCS). The architecture can serve as basis for developing a framework for identifying fault symptoms before it occurs.

The work involves techniques for managing uncertainty and predictive modeling of future behavior. The steering behavior depends on the velocity. We set the nominal velocity as reference. $50 \%$ of nominal velocity either side was set as understeering and oversteering. Several sensors were calibrated and compensated beforehand in order to measure the measurands such as angle, acceleration, yaw rate and normal velocity. As mentioned in the equation, the effect of instability scenario was also introduced in the modeling. The wheelchair's lane change behavior was studied multiple times. Similar to actuation valve, the residuals were generated and estimation was produced. Fig.8 shows the results corresponding to lane change behavior and its predictive model.

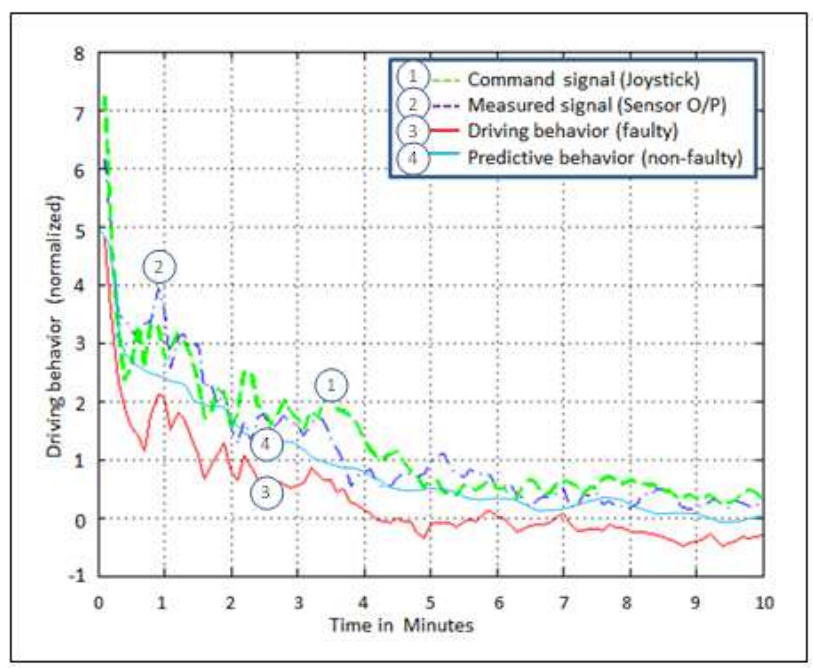

Figure 8. Results showing command signal (Joystick, measured signal (Sensor O/P), faulty driving behavior, and predictive driving behavior (non-faulty based on model based FDI).

\subsection{Spectral Analysis based FDI}

The wheelchair has many components responsible for its operation. The bottom part of the Fig. 6 depicts the movement of the joystick and the resulting output. One such essential component is the LVDT sensor which plays significant role in lane change behavior. It is an electromechanical transducer which is coupled to the wheelchair and it converts the joystick movement into the core movement. LVDT is encountered with different kind of fault, out of which the most common are overvoltage faults, core not moving fault and inductance or coil fault. Over- or under-voltage occurs when the applied voltage is in excess that cannot be handled by the primary coil of the LVDT.

Non-movement of core is associated with flux equivalency and corresponding differential voltages at both the coils. In this study, we have modeled the system where all these faults can have one index or signature that what we call inefficiency of the coil. The considered fault occurrence in an LVDT results from the inefficiency of the coils. It is assumed that the joystick makes slight deviations of about $8^{\circ}$ with respect to the expected line while moving along a straight path. Due to this core movement there is an angle involved which is detected with the help of an angle sensor. The output of an angle sensor is a random signal as shown in Fig.9(a). 
Scalogram of the non-faulty random signal we get is shown in Fig.9b.

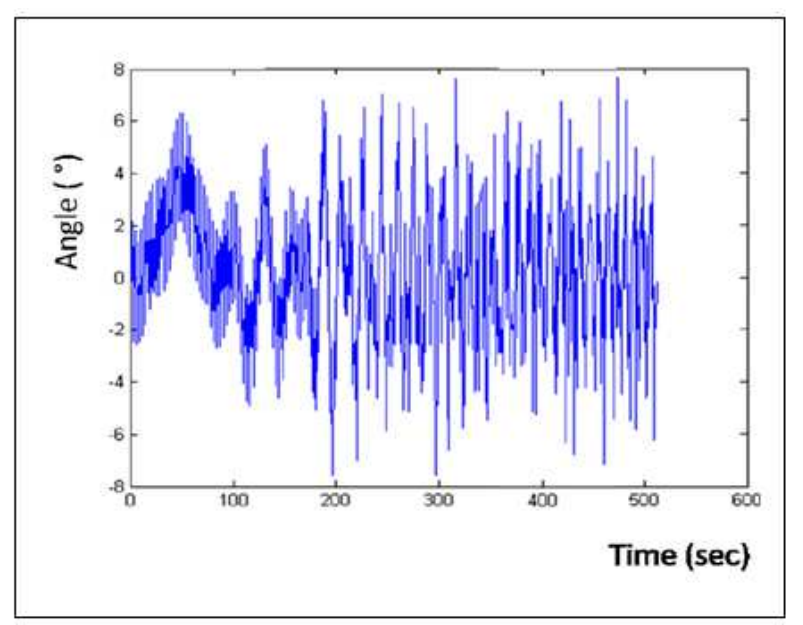

(a)

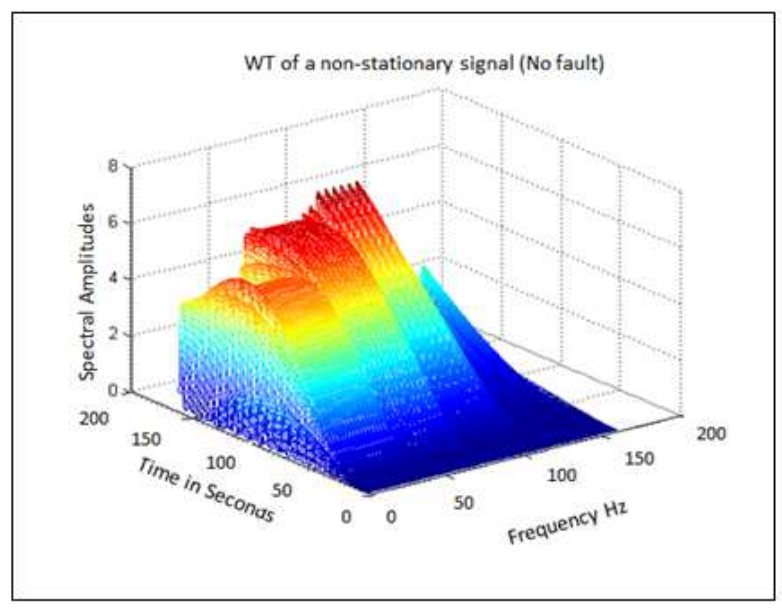

(b)

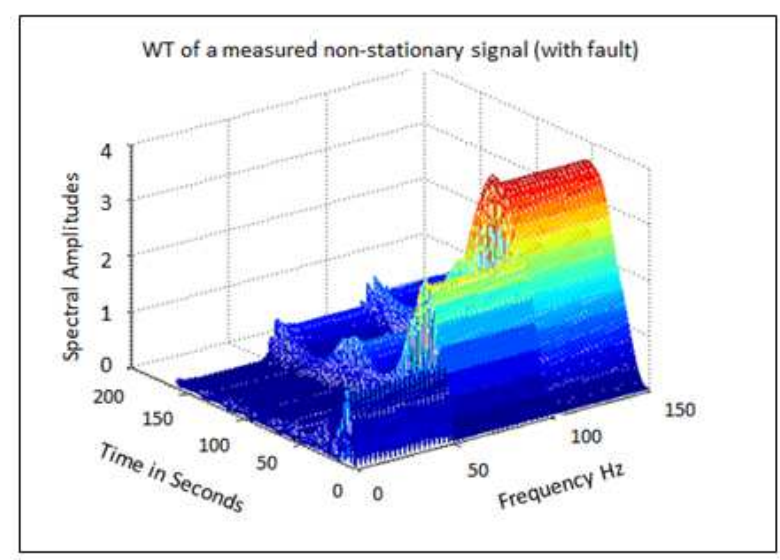

(c)

Figure 9. Results showing spectral analysis based FDI [(a) The random signal expected at the output of an angle sensor, (b) Scalogram of the LVDT signal showing all the frequency components, (c) Scalogram of the random signal obtained from the angle sensor output superimposed with the LVDT fault].
The fault in the core takes place when the command is given by the joystick but the relative movement of the core stops. In our simulation study the random signal from an angle sensor and the dither signal are superimposed and its WT is computed using the MATLAB. The Fig.9(c) shows the superimposition of the random signal obtained from an angle sensor output and the LVDT fault (core not moving). It is clear from the figure that the WT is able to record the change in the frequency components, as well as their time localization.

\section{Conclusions}

This paper provides validation of different FDI schemes based on the results obtained through several experimental studies [13]. The studies were carried out for model based feature estimation and spectral analysis based multiresolution signal decomposition methods, respectively. First the feature estimation based FDI for actuating valve driven by two motors was illustrated. In this case the experimental study was carried out for an electrical motor. Several experiments were conducted with actuator based valve control system for the validation of residual evaluation. As a second example, in this paper a rehabilitation wheel chair was considered for feature estimation based fault detection evaluation. The objective was to detect and isolate the faults in steering actuators. The classification and confirmation of the faults are implemented with the help of Artificial Neural Network (ANN) and Fuzzy Logic (FL) [14]. Finally, the validation of multiresolution technique of a sensor was demonstrated. The failure modes were considered. The scalograms of the output signals with the faults signatures were examined and detected.

\section{Acknowledgements}

The authors acknowledge Prof. R. Isernann for developing the model based approaches for several dynamical systems. The model-based work is based on much of Prof. R. Isernann's earlier work.

\section{References}

[1] Juan Dai, Chen, C.L.P., Xiao-Yan Xu, Peng Hu, Condition monitoring on complex machinery for predictive maintenance and process control, IEEE International Conference on Systems, Man and Cybernetics, 2008. SMC 2008., vol., no., pp.3595,3600, $12-15$ Oct. 2008

[2] Dogan Gökhan and Murat Başaran, Condition monitoring of speed controlled induction motors using wavelet packets and discriminant analysis, Journal of Expert Systems with Applications, Volume 38 Issue 7, pp 8079-8086, 2011, Pergamon Press, Inc. Tarrytown, NY, USA

[3] Rolf Isermann, Model-based fault-detection and diagnosis status and applications, Annual Review in Control, 29 (2005) $71-85$ 
[4] Mohanty, S.K.; Mahalik, N., "Some Studies on FDI in Actuating Systems," Process Automation, Control and Computing (PACC), 2011 International Conference on , vol., no., pp.1,6, 20-22 July 2011, doi: 10.1109/PACC.2011.5979009

[5] Mohanty, S, "Fault Detection in Mechatronic Systems", $\mathrm{PhD}$ Thesis, 2006, Utkal University, India

[6] Nadia Ben Amor, Multifont Arabic Characters Recognition Using Hough Transform and HMM/ANN Classification, Journal of multimedia, vol. 1, no. 2, May 2006

[7] Magdi. A. Koutb, M. Nabila, El-Rabaie, Hamdi. A. Awad, Ibrahim. A. Abd El-Hamid, Neural Fuzzy Fault Detection And Isolation In Greenhouses, IEEE Control System Magazine, pp. 28-47,

[8] Emmanuel Mazars, Imad M. Jaimoukha, and Zhenhai Li, Computation of a Reference Model for Robust Fault Detection and Isolation Residual Generation, Journal of Control Science and Engineering, 2008

[9] http://serdis.dis.ulpgc.es/ ii-rf/Manuales/Matlab/Matlab\%205 \%20-\%20Reference\%20Manual.PDF

[10] H. T. Mok, C. W. Chan, Online fault detection and isolation of nonlinear systems based on neuro-fuzzy networks, Engineering Applications of Artificial Intelligence, Vol. 21, Iss. 2, pp 171-181, 2008

[11] Inseok Hwang, Sungwan Kim, Youdan Kim and Chze Eng Seah, A Survey of Fault Detection, Isolation, and Reconfiguration Methods, IEEE Transaction on Control Systems Technology, Vol 18, No. 3, 2010.
[12] A. Ashokan and D. Sivakumar, Intergration of Fault Detection and Isolation Control In A Multi-Input Multi-Output System, Journal Automation \& System Engineering,

[13] D. Füssel and R. Isermann, Hierarchical motor diagnosis utilizing structural knowledge and a self-learning neuro-fuzzy-scheme. IEEE Trans. on Ind. Electronics, Vol. 74, No. 5, pp. 1070-1077, 2000

[14] S.M. El-Shal, A.S. Morris, A fuzzy expert system for fault detection in statistical process control of industrial processes, IEEE Transactions on Applications and Reviews, Systems, Man, and Cybernetics, Part C, Vol. 30, Iss. 2, pp. $281-289$, 2000

[15] Balle, P.; Isermann, Rolf, "Fault detection and isolation for nonlinear processes based on local linear fuzzy models and parameter estimation," American Control Conference, 1998. Proceedings of the 1998, vol.3, no., pp.1605,1609 vol.3, 21-26 Jun 1998, doi: 10.1109/ACC.1998.707277

[16] Jie Chen and R.J. Patton, "Robust Model-Based Fault Diagnosis for Dynamic Systems", Book, Springer Science Business Media, New York., 1999

[17] B. Freyermuth, R. Isermann (Ed.), "Fault Detection, Supervision and Safety for Technical Processes" IFAC Symposia Series, 1991.

[18] Harbilas Bal and N. P. Mahalik, "Fault detection (FDI) in actuators, 32nd Annual Central California Research Symposium, California State University, April 6, Fresno, California. 